\title{
Esperanza de Vida Ajustada por la Calidad en España: una aproximación
}

\section{Quality-Adjusted Life Expectancy in Spain: an estimate}

Núm. 6 (2016), pp. 28-48

Martínez Pérez, Jorge Eduardo*1

Sánchez Martínez, Fernando Ignacio ${ }^{*_{2}}$

Abellán Perpiñán, José María*3

Recibido: septiembre, 2015

Aceptado: noviembre, 2015

JEL Clasif: I15; I19;Joo

DOI: $10.5944 /$ reppp.6.2016.15348

\footnotetext{
*1 Jorge Eduardo Martínez Pérez. Universidad de Murcia. E-mail: jorgemp@um.es

*2 Fernando Ignacio Sánchez Martínez. Universidad de Murcia. E-mail: fernando@um.es

*3 José María Abellán Perpiñán. Universidad de Murcia. E-mail:dionisos@um.es
} 


\title{
Resumen
}

El objetivo de este trabajo es proporcionar una primera estimación de la Esperanza de Vida Ajustada por la Calidad para España. Esta medida sintética que combina cantidad y calidad de vida, puede ser un instrumento valioso para la evaluación de programas públicos destinados a aumentar la esperanza de vida y mejorar la calidad de vida de la población. Asimismo, esta estimación puede servir para validar las estimaciones previamente realizadas en España a escala territorial.

Palabras clave: Esperanza de Vida Ajustada por la Calidad; Calidad de Vida relacionada con la Salud; stock de capital salud

\begin{abstract}
This paper presents a first estimation of the Quality-Adjusted Life Expectancy (QALE) in Spain. The QALE combines length of life with quality of life and is intended to be a valuable instrument for the evaluation of public projects that are targeted to improve people's life expectancy and quality of life. Moreover, the estimated value for QALE in Spain may be useful to test the validity of previous estimations at a regional level.
\end{abstract}

Key Words: quality adjusted life expectancy; health-related quality of life; health capital 


\section{Introducción}

Son numerosos los programas y políticas públicas que tienen algún tipo de impacto sobre la mortalidad y morbilidad de la población: desde los proyectos de infraestructuras, hasta las políticas de seguridad y salud laboral, pasando por la política energética o, como es obvio, los programas de asistencia sanitaria y salud pública. La correcta evaluación del impacto de cualquiera de estos y otros programas y políticas exige cuantificar los costes (beneficios) en términos de pérdida (ganancia) de años de vida de la población, así como de deterioro (mejora) de su salud.

Hasta hace unas pocas décadas, el foco de la evaluación estuvo en la contribución de las políticas públicas al aumento de la supervivencia. A lo largo del siglo XX, sin embargo, las condiciones de vida y salubridad de la población española mejoraron de forma tal que la esperanza de vida al nacer se duplicó con creces entre 1900 y 2000, pasando de 34,76 a 79,34 años. Llegados al momento actual, en el que la esperanza de vida en España, como en el resto de países desarrollados, es elevada y cada vez resulta más difícil lograr incrementos adicionales en supervivencia, las condiciones de salud en las que los individuos disfrutan de este largo horizonte vital han pasado a ocupar un lugar prioritario en la agenda. En consecuencia, el objetivo de preservar la vida de los ciudadanos y prolongar su duración, está siendo progresivamente complementado, cuando no reemplazado, por el de mejorar la calidad (relacionada con la salud) de dicha vida.

Para evaluar el logro de este objetivo bidimensional, una vida más larga y con más salud, resulta necesario disponer de indicadores sintéticos que tengan en cuenta, no solo la supervivencia, sino también la incidencia de la morbilidad. Existen diferentes medidas que responden a esta caracterización, desde que Sanders (1964) y Sullivan (1971) realizaran las primeras aportaciones en este campo. Los instrumentos desarrollados inicialmente distinguían el «valor» de los años de vida disfrutados por un individuo atendiendo a una clasificación dicotómica (por ejemplo, sufrir una discapacidad frente a no padecerla, o disfrutar de buena salud frente a no hacerlo). La Esperanza de Vida Libre de Discapacidad o la Esperanza de vida en Buena Salud constituyen ejemplos de este tipo de instrumentos. Una segunda categoría de indicadores incorpora un conjunto más amplio de situaciones de salud posibles, a las que se asignan unos pesos de calidad de vida explícitos (también denominados «utilidades»), acotados por el valor máximo de 1, equivalente a la plena salud, y que reflejan la distancia que separa cada una de estas situaciones de la salud perfecta. El ejemplo típico de este segundo tipo de instrumentos es la Esperanza de Vida Ajustada por la Calidad (EVAC) (Fanshel y Bush, 1970; Wilkins y Adams, 1992, Murray et al., 2002), que informa sobre el número de Años de Vida Ajustados por la Calidad (AVAC) que se espera disfrute un individuo de una sociedad, a diferentes edades, asumiendo que su expectativa de supervivencia y su estado de salud futuro son iguales a los observados para la correspondiente cohorte de edad en el momento actual. Los AVAC combinan cantidad (tiempo) y calidad de vida en un único valor y constituyen una de las medidas de efectividad más utilizadas en la evaluación económica (análisis coste-utilidad) de tecnologías sanitarias. Para el cómputo de la EVAC se precisa disponer de información relativa, tanto a la supervivencia, input básico para el cómputo de la esperanza de vida, como a la calidad de vida relacionada con la salud (CVRS) que disfrutan los individuos que sobreviven. 
La EVAC es en un indicador especialmente valioso, en la medida en que es capaz de sintetizar en un único valor el comportamiento en términos de supervivencia y CVRS de la población. Su cómputo, así como el análisis de su evolución en el tiempo, pueden ser muy útiles para la evaluación de las políticas públicas. En España se dispone de algunas estimaciones de las medidas sintéticas citadas al comienzo del párrafo anterior (Esperanza de Vida Libre de Discapacidad y Esperanza de Vida en Buena Salud). ${ }^{1}$ Sin embargo, no existen estimaciones de la EVAC a escala nacional, debido a la ausencia, hasta fechas relativamente recientes, de información sobre CVRS de la población española susceptible de ser utilizada para su cálculo. ${ }^{2}$ Las encuestas regionales de salud en ciertas comunidades autónomas (como Cataluña o Andalucía) sí han recogido la información de base para obtener los pesos de calidad de vida que el cálculo de la EVAC requiere, de ahí que existan algunas estimaciones de este indicador a escala regional.

El presente trabajo pretende, por tanto, un doble objetivo: de un lado, proporcionar una primera estimación de la EVAC para el conjunto de España y, de otro, comparar los resultados obtenidos con los previamente estimados a escala autonómica con la intención de contrastar su validez. La estructura del artículo es como sigue: en el apartado siguiente se describen brevemente los antecedentes teóricos y empíricos; en el siguiente epígrafe se describe el origen y naturaleza de los datos utilizados, así como la metodología seguida para el cómputo de la EVAC; a continuación se da cuenta de los principales resultados del trabajo, que se cierra con una sección en la que se resumen las conclusiones del estudio y se reconocen las limitaciones del mismo, así como se sugieren futuras vías de investigación.

\section{Fundamentos y antecedentes}

Como se ha señalado en la introducción, el cálculo de la EVAC precisa de información relativa, tanto a la supervivencia a diferentes edades, como a la calidad de vida que se disfruta en cada una de ellas. Mientras que en relación con el primero de estos inputs, los datos de mortalidad/supervivencia, no existen problemas metodológicos ni de disponibilidad a escala regional, nacional e internacional, la medición de la CVRS plantea algunas cuestiones que resulta necesario puntualizar. En primer lugar, las medidas de CVRS se caracterizan por tres rasgos fundamentales (Badía y Lizán, 2003):

a) El carácter multidimensional del concepto, que engloba los dominios físico, psíquico y social.

b) El carácter subjetivo de la medición, que debe ser efectuada, por tanto, por el propio individuo.

c) La traslación de las respuestas a puntuaciones numéricas.

1 Véase Ministerio de Sanidad y Política Social (2009) y Ministerio de Sanidad, Servicios Sociales e Igualdad (2013)

$2 \quad$ La Encuesta Nacional de Salud 2011-12 (cuyos resultados se publicaron en 2013) fue la primera que recogió información sobre CVRS a través de un sistema descriptivo, el EQ-5D, que en principio permitiría la obtención de pesos de calidad de vida con los que ajustar la esperanza de vida de la población para estimar la EVAC. 
Dos son los posibles enfoques a la hora de llevar a cabo el proceso de valoración: de un lado, el enfoque de la psicología y, de otro, el de la economía de la salud. El primero pretende recoger cambios en la salud de un individuo o diferencias entre individuos mediante el empleo de cuestionarios en los que las respuestas se miden mediante escalas ordinales tipo Likert. El segundo enfoque, en cambio, utiliza medidas basadas en preferencias que procuran captar la intensidad de las mismas y, por ello, emplea escalas cardinales. Este segundo enfoque es el relevante para estimar la EVAC, pues las medidas de la CVRS no basadas en preferencias (enfoque psicométrico) tiene una serie de limitaciones, como pueden ser la imposibilidad de resumir en un único valor numérico el estado de salud del individuo o, caso de que ello fuera posible, el hecho de que dicho valor no se corresponda con una escala intervalo definida entre o y 1 (correspondiendo 1 a la salud plena y $\mathrm{o}$ a la muerte). ${ }^{3}$

Un ejemplo de las medidas procedentes del enfoque psicométrico es el cuestionario SF-36, una medida genérica de CVRS desarrollada en Estados Unidos (Ware y Sherbourne, 1992) para su empleo en la evaluación de tratamientos médicos. El SF-36 consta de 36 preguntas referidas a 8 dimensiones: Función Física, Rol Físico, Dolor Corporal, Salud General, Vitalidad, Función Social, Rol Emocional y Salud Mental. Las respuestas a cada una de las preguntas referidas a las diferentes dimensiones reciben una puntuación que, por agregación, da lugar a una valoración para cada una de las ocho dimensiones. Adicionalmente, la combinación de estos valores resumen de cada dimensión proporciona dos valoraciones globales: -Componente Resumen Mental y Componente Resumen Físico-. Sin embargo, el SF-36 no ofrece un único valor que resuma la calidad de vida del sujeto y, además, las puntuaciones globales no cumplen los requisitos de una escala intervalo, tal y como se ha señalado antes.

Entre las medidas de CVRS basadas en preferencias cabe citar el EQ-5D, el Health Utilities Index (HUI) o el SF-6D. Esta última resulta de particular interés por tratarse de una extracción del cuestionario SF-36, reseñado en el párrafo anterior y que es ampliamente conocido y utilizado en el ámbito clínico. El SF-6D (Brazier et al., 1998; 2002) resume las respuestas al SF-36 en un sistema descriptivo que considera 6 dimensiones, cada una de las cuales puede admitir un número variable de niveles de gravedad, según el sujeto no sufra ningún problema en una determinada dimensión de la salud (nivel 1) o sufra muchos o muy graves problemas en ella (nivel 4, 506 , dependiendo de la dimensión de que se trate). El sistema descriptivo se resume en el cuadro 1 y con él es posible caracterizar un total de 18.000 estados diferentes.

3 En una escala intervalo, a diferencia de lo que ocurre con una escala meramente ordinal, la distancia entre los valores indica la magnitud (la intensidad) de las diferencias. Una escala ordinal indica si un objeto, situación, estado, etc. se valora más o menos que otro, pero no cuánto más o menos se valora. 
Cuadro I. El Sistema de clasificación de estados de salud SF-6D

\begin{tabular}{|c|c|c|c|c|c|c|}
\hline Nivel & $\begin{array}{c}\text { Funcionamiento } \\
\text { físico }\end{array}$ & $\begin{array}{c}\text { Limitaciones } \\
\text { en el rol }\end{array}$ & $\begin{array}{c}\text { Funcionamiento } \\
\text { social }\end{array}$ & Dolor & $\begin{array}{l}\text { Salud } \\
\text { mental }\end{array}$ & Vitalidad \\
\hline I & $\begin{array}{l}\text { Su salud no } \\
\text { le limita para } \\
\text { realizar esfuerzos } \\
\text { intensos (ej. correr, } \\
\text { levantar objetos } \\
\text { pesados, deportes } \\
\text { agotadores). }\end{array}$ & $\begin{array}{l}\text { No tiene } \\
\text { problemas con } \\
\text { su trabajo u } \\
\text {; otras actividades } \\
\text { cotidianas a } \\
\text { causa de su } \\
\text { salud física o de } \\
\text { sus problemas } \\
\text { emocionales. }\end{array}$ & $\begin{array}{l}\text { Su salud no le } \\
\text { dificulta sus } \\
\text { actividades } \\
\text { sociales (como } \\
\text { visitar a amigos } \\
\text { o familiares) en } \\
\text { ningún momento. }\end{array}$ & No tiene dolor. & $\begin{array}{l}\text { Nunca se } \\
\text { siente muy } \\
\text { nervioso o } \\
\text { desanimado y } \\
\text { deprimido. }\end{array}$ & $\begin{array}{l}\text { Tiene mucha } \\
\text { energía } \\
\text { siempre. }\end{array}$ \\
\hline 2 & $\begin{array}{l}\text { Su salud le limita } \\
\text { un poco para } \\
\text { realizar esfuerzos } \\
\text { intensos (p.ej. } \\
\text { correr, levantar } \\
\text { objetos pesados, } \\
\text { deportes } \\
\text { agotadores). }\end{array}$ & $\begin{array}{l}\text { Ha dejado de } \\
\text { hacer algunas } \\
\text { tareas en su } \\
\text { trabajo o en } \\
\text { sus actividades } \\
\text { cotidianas a causa } \\
\text { de su salud física. }\end{array}$ & $\begin{array}{l}\text { Su salud le dificulta } \\
\text { sus actividades } \\
\text { sociales (como } \\
\text { visitar a amigos } \\
\text { o familiares) solo } \\
\text { alguna vez. }\end{array}$ & $\begin{array}{l}\text { Tiene dolor pero } \\
\text { no interfiere con } \\
\text { su trabajo habitual } \\
\text { (fuera de casa o } \\
\text { en las tareas del } \\
\text { hogar). }\end{array}$ & $\begin{array}{l}\text { Se siente muy } \\
\text { nervioso o } \\
\text { desanimado } \\
\text { y deprimido } \\
\text { solo alguna } \\
\text { vez. }\end{array}$ & $\begin{array}{l}\text { Tiene mucha } \\
\text { energía casi } \\
\text { siempre. }\end{array}$ \\
\hline 3 & $\begin{array}{l}\text { Su salud le limita } \\
\text { un poco para } \\
\text { realizar esfuerzos } \\
\text { moderados (ej. } \\
\text { mover una mesa, } \\
\text { pasar la aspiradora } \\
\text { o caminar más de } \\
\text { Ih). }\end{array}$ & $\begin{array}{l}\text { Hace menos de } \\
\text { lo que quisiera } \\
\text { hacer a causa de } \\
\text { sus problemas } \\
\text { emocionales. }\end{array}$ & $\begin{array}{l}\text { Su salud le dificulta } \\
\text { sus actividades } \\
\text { sociales (como } \\
\text { visitar a amigos o } \\
\text { familiares) algunas } \\
\text { veces. }\end{array}$ & $\begin{array}{l}\text { Tiene dolor que } \\
\text { interfiere un poco } \\
\text { con su trabajo } \\
\text { habitual (fuera } \\
\text { de casa o en las } \\
\text { tareas del hogar). }\end{array}$ & $\begin{array}{l}\text { Se siente muy } \\
\text { nervioso o } \\
\text { desanimado } \\
\text { y deprimido } \\
\text { algunas veces. }\end{array}$ & $\begin{array}{l}\text { Tiene mucha } \\
\text { energía } \\
\text { algunas } \\
\text { veces. }\end{array}$ \\
\hline 4 & $\begin{array}{l}\text { Su salud le limita } \\
\text { mucho para } \\
\text { realizar esfuerzos } \\
\text { moderados (p.ej. } \\
\text { mover una mesa, } \\
\text { pasar la aspiradora } \\
\text { o caminar más de } \\
\text { I hora). }\end{array}$ & $\begin{array}{l}\text { Ha dejado de } \\
\text { hacer algunas } \\
\text { tareas en su } \\
\text { trabajo o en } \\
\text { sus actividades } \\
\text { cotidianas por su } \\
\text { salud física y hace } \\
\text { menos de lo que } \\
\text { quisiera a causa } \\
\text { de sus problemas } \\
\text { emocionales. }\end{array}$ & $\begin{array}{l}\text { Su salud le dificulta } \\
\text { sus actividades } \\
\text { sociales (como } \\
\text { visitar a amigos } \\
\text { o familiares) casi } \\
\text { siempre. }\end{array}$ & $\begin{array}{l}\text { Tiene dolor } \\
\text { que interfiere } \\
\text { moderadamente } \\
\text { con su trabajo } \\
\text { habitual (fuera } \\
\text { de casa o en las } \\
\text { tareas del hogar). }\end{array}$ & $\begin{array}{l}\text { Se siente muy } \\
\text { nervioso o } \\
\text { desanimado y } \\
\text { deprimido casi } \\
\text { siempre. }\end{array}$ & $\begin{array}{l}\text { Tiene mucha } \\
\text { energía solo } \\
\text { alguna vez. }\end{array}$ \\
\hline 5 & $\begin{array}{l}\text { Su salud le limita } \\
\text { un poco para } \\
\text { bañarse o vestirse } \\
\text { por sí mismo. }\end{array}$ & & $\begin{array}{l}\text { Su salud le dificulta } \\
\text { sus actividades } \\
\text { sociales siempre } \\
\text { (ej. visitar a amigos } \\
\text { o familiares). }\end{array}$ & $\begin{array}{l}\text { Tiene dolor que } \\
\text { interfiere bastante } \\
\text { con su trabajo } \\
\text { habitual (fuera de } \\
\text { casa o en tareas } \\
\text { del hogar). }\end{array}$ & $\begin{array}{l}\text { Se siente muy } \\
\text { nervioso o } \\
\text { desanimado } \\
\text { y deprimido } \\
\text { siempre. }\end{array}$ & $\begin{array}{l}\text { Nunca } \\
\text { tiene mucha } \\
\text { energía. }\end{array}$ \\
\hline 6 & $\begin{array}{l}\text { Su salud le limita } \\
\text { mucho bañarse/ } \\
\text { vestirse por sí } \\
\text { mismo. }\end{array}$ & & & $\begin{array}{l}\text { Tiene dolor que } \\
\text { interfiere mucho } \\
\text { con su trabajo } \\
\text { habitual. }\end{array}$ & & \\
\hline
\end{tabular}

Fuente: Elaboración propia a partir de Brazier et al. (2002). 
La traducción de un determinado estado de salud descrito mediante un sistema como el SF-6D, el EQ-5D o el HUI a un valor que refleje el peso de calidad de vida en una escala o-1 se realiza mediante la aplicación de un algoritmo con el que se genera la «tarifa» o conjunto de utilidades asociado a cada instrumento. ${ }^{4}$ Los parámetros de estos algoritmos se estiman mediante técnicas econométricas (análisis de regresión) a partir de valoraciones obtenidas en encuestas a población general. En el cuadro 2 se muestran los algoritmos de cálculo de las utilidades asociadas al instrumento SF-6D en el Reino Unido (Brazier y Roberts, 2004) ${ }^{5}$ y en España (Abellán et al., 2012a). Siendo 1 el valor asociado a la salud plena, los coeficientes que aparecen en las columnas indican el valor que hay que restar a la unidad en función del nivel de gravedad registrado en cada una de las 6 dimensiones.

Cuadro 2. Algoritmos de cálculo de las utilidades (pesos de calidad de vida) asociados al sistema descriptivo SF-6D (derivado del SF-36) en el Reino Unido y España.

\begin{tabular}{|c|c|c|}
\hline Dimensión/Nivel & Brazier y Roberts (2004) ${ }^{\text {(a) }}$ & Abellán et al $(20 \text { I } 2 a)^{(b)}$ \\
\hline Función Física 2 & \multirow{2}{*}{-0.035} & -0.150 \\
\hline Función Física 3 & & -0.034 \\
\hline Función Física 4 & -0.044 & -0.090 \\
\hline Función Física 5 & -0.056 & -0.111 \\
\hline Función Física 6 & -0.117 & -0.338 \\
\hline Limitaciones en el rol 2 & \multirow{3}{*}{-0.053} & -0.014 \\
\hline Limitaciones en el rol 3 & & -0.038 \\
\hline Limitaciones en el rol 4 & & -0.070 \\
\hline Funcionamiento Social 2 & -0.057 & -0.037 \\
\hline Funcionamiento Social 3 & -0.059 & -0.060 \\
\hline Funcionamiento Social 4 & -0.072 & -0.203 \\
\hline Funcionamiento Social 5 & -0.087 & -0.208 \\
\hline Dolor 2 & \multirow{2}{*}{-0.042} & -0.018 \\
\hline Dolor 3 & & -0.034 \\
\hline Dolor 4 & -0.065 & -0.198 \\
\hline Dolor 5 & -0.102 & -0.202 \\
\hline Dolor 6 & -0.171 & -0.318 \\
\hline Salud Mental 2 & \multirow{2}{*}{-0.042} & -0.066 \\
\hline Salud Mental 3 & & -0.078 \\
\hline Salud Mental 4 & -0.100 & -0.096 \\
\hline Salud Mental 5 & -0.118 & -0.224 \\
\hline Vitalidad 2 & \multirow{3}{*}{-0.07 I } & -0.058 \\
\hline Vitalidad 3 & & -0.121 \\
\hline Vitalidad 4 & & -0.157 \\
\hline Vitalidad 5 & -0.092 & -0.199 \\
\hline MOST (*) & -0.061 & --- \\
\hline
\end{tabular}

(a) Modelo de medias (2) de la Tabla 4 de Brazier y Roberts (2004). En algunas dimensiones existe un único coeficiente para dos o más niveles, debido a que los coeficientes estimados daban lugar a inconsistencias.

(b) Modelo de medias recomendado.

(*) Pérdida de utilidad adicional en la tarifa británica cuando en, al menos, una de las dimensiones se registra el nivel máximo de gravedad $(4,5$ ○ 6 , según el caso).

Fuente: Elaboración propia a partir de Brazier y Roberts (2004) y Abellán et al. (20I2a).

4 Los valores pueden resultar negativos, lo que se interpreta como que los estados de salud en cuestión se consideran «peores que la muerte» (a la que se asigna el valor 0 ).

5 Aunque el algoritmo del SF-6D basado en el SF-36 fue inicialmente estimado en Brazier et al. (2002), en Brazier y Roberts (2004) se presenta una tarifa «consistente» para el SF-6D/SF-36 que mejora la estimación inicial y es la recomendada por los autores. 
Como se señaló en la introducción, existen estimaciones previas de la EVAC en nuestro país, si bien la práctica totalidad de ellas se ha llevado a cabo a escala regional. Buena parte de estas estimaciones han tenido, además, un carácter instrumental, en la medida en que se han utilizado como una etapa intermedia en la estimación del valor monetario del stock de salud. ${ }^{6}$ Curiosamente, el primer intento de valorar el stock de salud a partir de una estimación de la EVAC es el único cuyo ámbito territorial es el conjunto de España y se debe a García-Altés et al. (2006). En su trabajo, los pesos de calidad de vida se derivaron a partir de dos resultados de la Encuesta Nacional de Salud: las respuestas a la pregunta subjetiva sobre el estado de salud y la situación objetiva, en términos de dolencias crónicas, declarada por los entrevistados.

La inclusión de cuestionarios de CVRS en las encuestas regionales de salud ha permitido la estimación de la EVAC en algunas comunidades autónomas españolas. Zozaya et al. (2005) utilizaron datos de la Encuesta de Salud de Cataluña de 1994 y 2002 y, más tarde, Oliva (2008) empleó también la oleada de 2006. Esta encuesta incluye un «autoclasificador» del instrumento EQ-5 $\mathrm{D}^{7}$, a partir del cual se pueden obtener pesos de calidad de vida de manera análoga a la expuesta para el SF-6D, utilizando a tal fin la tarifa española de dicho instrumento (Badía et al. 1995; 2001). Tras combinar los datos de calidad de vida con los de esperanza de vida, los autores concluyeron que el stock de salud en Cataluña se había deteriorado en el período objeto de estudio. Oliva y Zozaya (2007) llevaron a cabo un análisis similar en 2004 utilizando información de la Encuesta de Salud de Canarias que, al igual que la catalana, incluía el instrumento EQ-5D. La comparación de la EVAC estimada para las Islas Canarias en 2004 con la estimación realizada en Cataluña da como resultado valores inferiores en Canarias para la mayoría de los grupos de edad considerados, con la excepción de las mujeres en los tramos de mayor edad. ${ }^{8}$

Esnaola et al. (2006) estimaron la EVAC del País Vasco en 1997 y 2002 utilizando en este caso como instrumento de CVRS el SF-6D en lugar del EQ-5D, toda vez que la encuesta de salud vasca contiene el cuestionario SF-36, del que se deriva, como se ha dicho, el sistema SF-6D. En ausencia de un algoritmo basado en preferencias de la población española con el que obtener los pesos de calidad de vida, los autores aplicaron la tarifa británica (Brazier et al., 2002). Las principales conclusiones del estudio fueron que la EVAC no se había alterado significativamente en el quinquenio objeto de consideración, y que su valor estimado resultaba ser superior al de otros países como Portugal y Reino Unido. Una metodología similar fue empleada por Martínez et al. (2008), con información extraída de la Encuesta Andaluza de Salud del año 2003. La principal diferencia respecto al trabajo de Esnaola et al. (2006) estriba en el hecho de que el instrumento

$6 \quad$ El valor monetario del stock de salud se obtiene como el producto de una variable que mide cantidades (la EVAC), y otra que recoge precios (el valor monetario del AVAC). Los trabajos pioneros en este campo tienen lugar en Estados Unidos, donde Cutler y Richardson $(1997,1998,1999)$ definen el concepto como el producto entre el valor presente descontado del número de AVAC que un individuo se espera que disfrute a lo largo de su vida, dadas las condiciones de supervivencia y salud actualmente observadas, y el valor monetario que esa sociedad atribuye

$7 \quad$ El EQ-5D describe el estado de salud mediante 5 dimensiones: movilidad; autocuidado; actividades cotidianas; dolor/malestar; ansiedad/depresión. En la versión utilizada en los estudios que se citan en este párrafo, cada una de la dimensiones puede tomar 3 niveles, según la intensidad/gravedad de los problemas, lo que da lugar a 243 estados posibles.

8 Una explicación de las diferencias en CVRS entre las poblaciones residentes en las Islas Canarias y en Cataluña puede encontrarse en Oliva et al. (2010). 
genérico incluido en la encuesta andaluza no era el SF-36, sino el SF-12 (una versión reducida del primero). Por esta razón, el algoritmo aplicado para la obtención de los pesos de calidad de vida fue uno distinto al mostrado en el cuadro 2, si bien igualmente estimado a partir de las preferencias de la población británica (Brazier y Roberts, 2004). Los resultados de este estudio revelan la importancia de las diferencias en calidad de vida por sexos, así como el hecho de que los valores obtenidos, con la limitaciones propias de la comparabilidad, no difieren sustancialmente de los estudios previos realizados en España (por ejemplo, el ya citado del País Vasco). Finalmente, Abellán et al. (2012b), realizaron una estimación del valor del stock de capital salud en la Región de Murcia, a partir de una encuesta propia, con una muestra representativa por sexo y cuotas de edad de la población adulta de la comunidad autónoma, a la que se administró el cuestionario SF-36. La EVAC se estimó aplicando las tarifas británica (Brazier y Roberts, 2004) y española (Abellán et al., 2012a) del SF-6D, obteniendo unos resultados que no diferían mucho de estimaciones previas para otros territorios.

\section{Material y métodos}

La información demográfica básica para el cómputo de la EVAC, esto es, la relativa al tamaño de los grupos por edad y sexo y la mortalidad que permiten el cómputo de la esperanza de vida por edades, se encuentra disponible en las Tablas de Mortalidad confeccionadas por el Instituto Nacional de Estadística. En lo concerniente a la CVRS para cada uno de estos grupos, se ha de recurrir a alguna fuente que ofrezca datos sobre el estado de salud de la población española, desagregados por edad y sexo, y recogidos mediante alguno de los sistemas descriptivos a los que se hizo alusión en el apartado segundo: EQ-5D, SF-6D, HUI, ... La opción, en principio, más plausible sería la de acudir a la Encuesta Nacional de Salud de España (ENS) 2011-12 que, por primera vez, incluyó el EQ-5D para caracterizar el estado de salud de los encuestados, lo que en principio permitiría obtener las utilidades o pesos de calidad de vida. Sin embargo, la versión del EQ-5D que contiene la ENS es diferente a la que se ha mencionado con anterioridad en este artículo, pues a diferencia de aquella, que distinguía 3 niveles de gravedad para cada dimensión (EQ-5D-3L) ésta distingue 5 niveles en cada atributo (EQ- $5 \mathrm{D}-5 \mathrm{~L}$ ). Este hecho limitaría las posibilidades de comparar los resultados que se obtuvieran con los de estudios previos a escala regional. Por otra parte, la nueva versión con 5 niveles del EQ-5D no disponía hasta fechas muy recientes de un algoritmo con el cual calcular las utilidades o pesos de calidad de vida, sino únicamente un instrumento provisional de conversión («crosswalk») que toma como referencia la tarifa del sistema con 3 niveles. ${ }^{9}$

En el presente estudio hemos optado por el instrumento SF-6D, que se describió en detalle en el cuadro 1, por su mayor sensibilidad para captar diferencias entre estados de salud relativamente leves -los más frecuentes en el caso de la población general-,

9 Recientemente, Ramos-Goñi, et al. (2015) (Postprint) han llevado a cabo un estudio de valoración con una muestra de 1000 sujetos, representativa de la población adulta española con la que han derivado un algoritmo para el EQ-5D-5L que asigna valores de utilidad más altos, en general, y menor frecuencia de valores negativos (estados «peores que la muerte») que los observados en la tarifa española de su predecesor el EQ-5D-3L. 
en oposición al EQ-5D, instrumento que adolece de este problema de incapacidad para discriminar entre problemas de salud leves conocido como efecto «techo» (Brazier et al., 2004)..$^{10}$ Asimismo, el SF-6D facilita una comparación más homogénea de nuestros resultados con tres de las estimaciones de la EVAC previamente obtenidas a escala territorial en Andalucía, País Vasco y la Región de Murcia, todas ellas basadas en los pesos de calidad de vida derivados a partir de dicho instrumento. La caracterización de la salud de la población española mediante el SF-6D se ha extraído de una encuesta representativa de la población adulta española, con un amplio tamaño muestral $(n=4.036)$, realizada por la Dirección General de Tráfico con fines diferentes a los de este estudio. ${ }^{11}$ La muestra es fruto de un muestreo polietápico estratificado por comunidad autónoma y tamaño de hábitat, habiéndose establecido, además, cuotas por tamaño de hábitat y comunidad autónoma, así como por grupos de edad y sexo. La encuesta personal, asistida por ordenador, incluía entre otras preguntas, el cuestionario SF-36, a partir del cual, y tras convertir sus respuestas al sistema descriptivo SF-6D, y aplicar el algoritmo estimado para España por Abellán et al. (2012a), se obtuvieron los pesos de calidad de vida o utilidades necesarios para el cómputo de la EVAC. Adicionalmente, la EVAC se ha estimado con los pesos de calidad de vida resultantes de aplicar la tarifa del SF-6D estimada por Brazier y Roberts (2004) para el Reino Unido, en aras de la homogeneidad del ejercicio de comparación con estudios previos que utilizaron el algoritmo británico.

Para el cómputo de la EVAC se parte de la información contenida en las Tablas de Vida que ofrecen, para cada edad, información relativa al tamaño del grupo y al número de años-persona en el intervalo de edad que va de $x$ a $x+n\left(L_{x}\right)$. A partir de las tablas de vida se calcula la Esperanza de Vida, utilizando la expresión siguiente:

$$
E V=\frac{\sum_{X}^{n} L_{x}}{l_{n}}
$$

El ajuste por calidad necesario para estimar la EVAC se realiza siguiendo la propuesta de Sullivan (1971), si bien, en lugar de emplear como factor corrector la prevalencia observada de una determinada dolencia, en nuestro caso utilizaremos los pesos de calidad de vida (utilidades) específicos para cada grupo de edad y sexo extraídos de la muestra de población general. Dichas utilidades ajustarán el número de años de vida vivido en cada intervalo de edad $\left(L_{x}\right)$ en función de su CVRS $\left(U_{x}\right)$, resultando la siguiente expresión:

$$
E V A C=\frac{\sum_{X}^{n} U_{x} L_{x}}{l_{n}}
$$

\footnotetext{
10 La versión de 5 niveles (EQ-5D-5L) reduce parcialmente el efecto «techo» y mejora la capacidad discriminatoria del instrumento, aunque está lejos de solucionar completamente los problemas mencionados (Janssen et al. 2013).

11 Los objetivos de este proyecto fueron estimar el «Valor de la Vida Estadística» en España, así como el valor monetario de los accidentes no mortales (véase Dirección General de Tráfico 2010, 2011; Martínez et al., 2015). Los autores cuentan con la autorización de la DGT para la explotación de los datos con fines académicos y tienen acceso a ellos por haber formado parte del equipo investigador del citado proyecto. Los investigadores interesados pueden contactar con los autores para acceder a la base de datos, previa autorización de su uso por parte de la DGT.
} 
Los valores de las utilidades que se emplean en la expresión (2) no son directamente los valores medios observados en la muestra, sino que previamente se ha aplicado un procedimiento de suavizado. La razón para ello es el reducido número de observaciones disponible para ciertos grupos de edad/sexo, lo que redundaba en valores poco plausibles. La estrategia que se ha seguido es la de aplicar un procedimiento kernel polinómico $^{12}$, utilizando, de entre las posibles aproximaciones según el grado del polinomio, la que garantiza la minimización de los errores en términos cuadráticos, siempre que se cumpla la condición de que la utilidad sea decreciente con la edad. Adicionalmente, se imputa a los grupos de edad por debajo de 18 años la misma calidad de vida que a los sujetos de dicha edad, al tiempo que se asume que, más allá de los 80 años la calidad de vida se mantiene constante. Ambos supuestos obedecen a que no se cuenta con información suficiente para ninguno de estos dos grupos de edad.

\section{Resultados}

El estado de salud de la población española, según las respuestas de los sujetos de la muestra al cuestionario SF-36 (y su posterior traducción al sistema descriptivo SF-6D), se caracteriza tal y como se resume en el Cuadro 3, en el que se identifican los estados de salud más frecuentes en la muestra. Un 19\% de los sujetos declara no tener ningún tipo de problema en cada una de las seis dimensiones que contiene el instrumento SF-6D, si bien la proporción de individuos cuya salud responde al estado 111111 supera el 22\% en el caso de los hombres y se limita al $15 \%$ en el caso de las mujeres. En total han sido necesarios 603 estados de salud distintos para describir la situación de salud de los integrantes de la muestra. También en este caso hay diferencias por sexos, toda vez que para categorizar la salud de las mujeres se ha precisado de un total de 411 estados, mientras que para los varones ha bastado con 343 .

Cuadro 3. Estados de salud descritos según el SF-6D más frecuentes en la muestra $(n=4036)$.

\begin{tabular}{|c|c|c|c|c|c|}
\hline Estado de Salud & Obs & Porcentaje & Estado de Salud & Obs & Porcentaje \\
\hline IIIIII & 766 & 18.98 & $2 I I I I$ & 46 & 1.14 \\
\hline IIIII22 & 507 & 12.56 & 211212 & 42 & 1.04 \\
\hline 111112 & 414 & 10.26 & 211123 & 37 & 0.92 \\
\hline|||||| $\mid$ & 219 & 5.43 & ||$|22|$ & 35 & 0.87 \\
\hline 111222 & 99 & 2.45 & 211223 & 34 & 0.84 \\
\hline 211122 & 98 & 2.43 & ||$|2| \mid$ & 30 & 0.74 \\
\hline 111123 & 94 & 2.33 & 111133 & 29 & 0.72 \\
\hline $1|I| I 3$ & 79 & 1.96 & 211113 & 26 & 0.64 \\
\hline 211112 & 71 & 1.76 & 222222 & 26 & 0.64 \\
\hline 211222 & 57 & $1.4 \mathrm{I}$ & I I I 223 & 25 & 0.62 \\
\hline 111212 & 51 & 1.26 & ||||$|3|$ & 22 & 0.55 \\
\hline 111132 & 49 & 1.21 & Resto de estados & 1180 & 29.24 \\
\hline
\end{tabular}

12 El procedimiento consiste básicamente en una regresión local polinomial y constituye una generalización del suavizado local de medias propuesto por Nadaraya (1964) y Watson (1964). Esta forma de actuar es computacionalmente más intensiva, pero goza de mejores propiedades estadísticas. Para una explicación en profundidad, véase Gutiérrez et al. (2003). 
En el Gráfico 1 se representa la esperanza de vida por edades y según sexo, calculada a partir de las Tablas de vida publicadas por el INE. La esperanza de vida al nacer se sitúa, para el conjunto de la población, en 81,9 años. Por sexos, es de 78,9 años para los varones y de 84,9 años para las mujeres, constatándose la existencia de una brecha significativa. Esta diferencia de 6 años en la esperanza de vida se va reduciendo lentamente conforme avanzamos en la estructura de edad, de tal forma que a los 40 años se ha reducido a 2,7 años pero a los 60 continúa por encima de los 2 años. No es hasta los 80 años cuando la diferencia en esperanza de vida entre hombres y mujeres empieza a ser de escasa magnitud (o,7 años), invirtiéndose el signo del diferencial a partir de los 95 años (con todas las cautelas que exige el reducido tamaño de la muestra en estos grupos de edad).

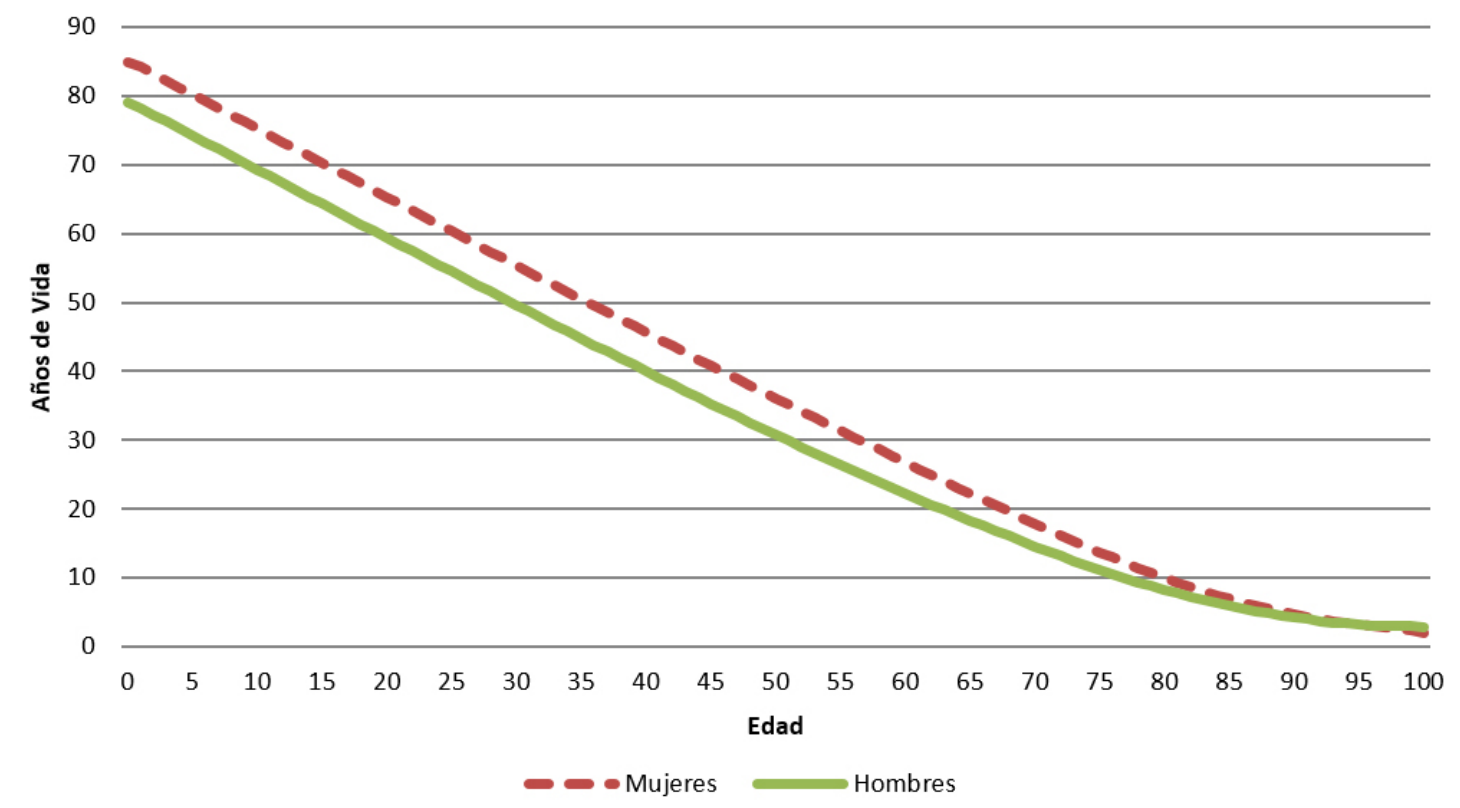

Gráfico I. Esperanza de vida por sexo y edad.

La información sobre calidad de vida extraída de la muestra, tanto la directamente observada (valores medios por edades), como la resultante del suavizado a través del procedimiento de kernel polinómico, se muestra en el Gráfico 2 para hombres y mujeres, por separado. Para el suavizado se eligió finalmente un polinomio de grado dos, por ser el que se comportaba mejor en términos de ajuste, sin contravenir la condición de que la utilidad fuese decreciente con la edad, como se observa en los gráficos. 

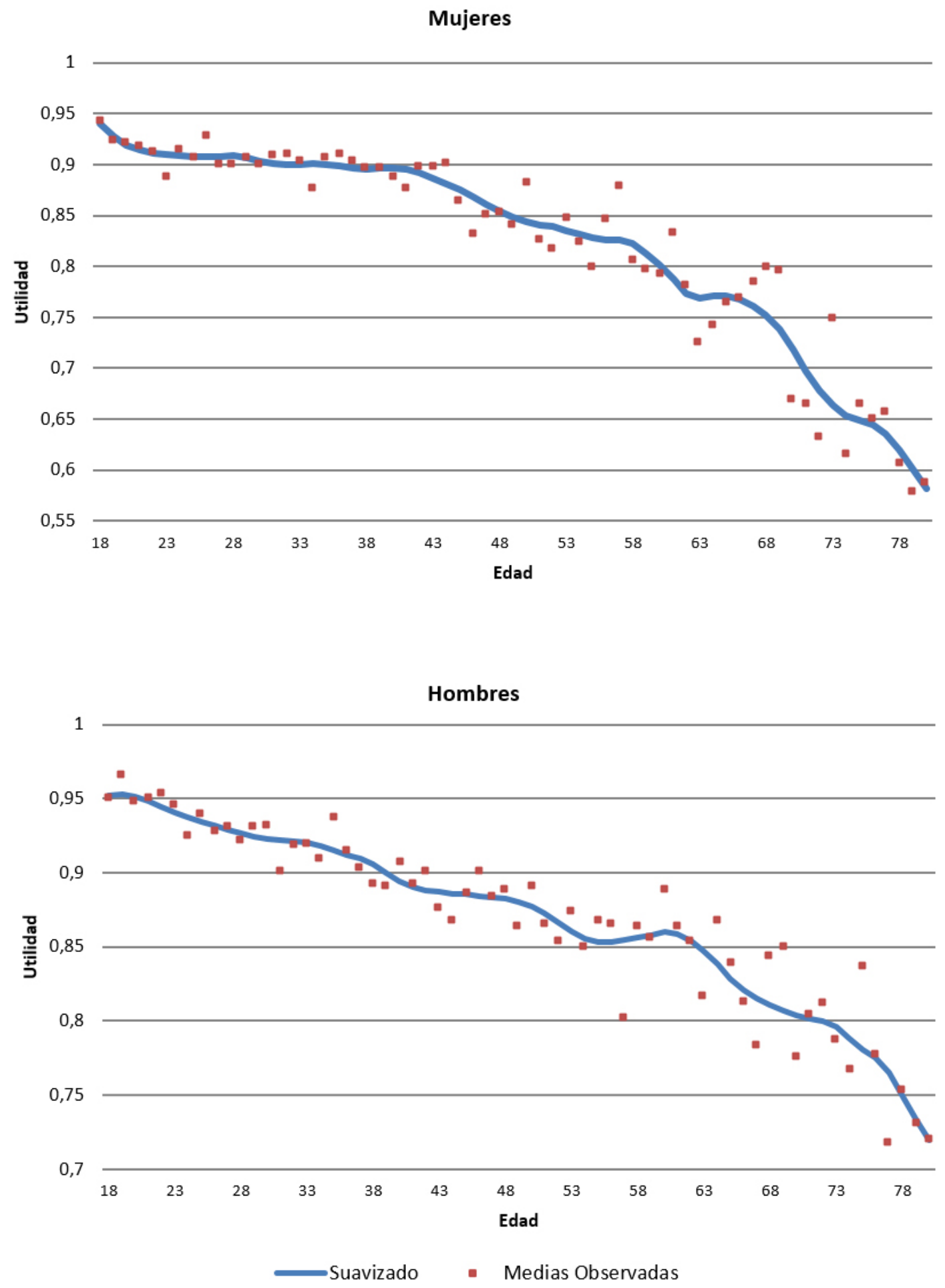

Gráfico 2. Utilidades medias observadas en la muestra y valores suavizados por sexo y edad. 
La combinación de los datos sobre esperanza de vida y calidad de vida según la expresión (2) anterior permite la estimación de la EVAC, cuyos resultados por sexo y edad se muestran en el Cuadro 4 y el Gráfico 3. El rasgo más sobresaliente de estos perfiles de EVAC, al ser comparados con los valores de esperanza de vida sin ajustar que se mostraron en el Gráfico 1, es la considerable reducción en la brecha por sexos. El mayor horizonte vital de las mujeres se ve compensado por una menor calidad de vida, prácticamente a todas las edades, de tal forma que, al combinar ambas dimensiones, las diferencias prácticamente se desvanecen. La EVAC al nacer para las mujeres asciende a poco más de 70,5 años de vida ajustados por la calidad (AVAC), mientras que en el caso de los varones dicho valor es de 69,9 AVAC.

Cuadro 4. Esperanza de vida ajustada por la calidad para España en 20II, por sexo y edad.

\begin{tabular}{|c|c|c|c|}
\hline Edad & Mujeres & Hombres & Total \\
\hline $\mathbf{0}$ & 70,5 & 69,9 & 70,4 \\
$\mathbf{I}$ & 69,8 & 69,1 & 69,7 \\
$\mathbf{2}$ & 68,9 & 68,2 & 68,8 \\
$\mathbf{3}$ & 68,0 & 67,3 & 67,8 \\
$\mathbf{4}$ & 67,0 & 66,3 & 66,9 \\
$\mathbf{5}$ & 66,1 & 65,4 & 66,0 \\
$\mathbf{6}$ & 65,2 & 64,4 & 65,0 \\
$\mathbf{7}$ & 64,3 & 63,5 & 64,1 \\
$\mathbf{8}$ & 63,3 & 62,5 & 63,1 \\
$\mathbf{9}$ & 62,4 & 61,6 & 62,2 \\
$\mathbf{1 0}$ & 61,5 & 60,6 & 61,3 \\
$\mathbf{1 1}$ & 60,5 & 59,7 & 60,3 \\
$\mathbf{1 2}$ & 59,6 & 58,7 & 59,4 \\
$\mathbf{1 3}$ & 58,7 & 57,8 & 58,4 \\
$\mathbf{1 4}$ & 57,7 & 56,8 & 57,5 \\
$\mathbf{1 5}$ & 56,8 & 55,9 & 56,6 \\
$\mathbf{1 6}$ & 55,9 & 54,9 & 55,6 \\
$\mathbf{1 7}$ & 54,9 & 54,0 & 54,7 \\
$\mathbf{1 8}$ & 54,0 & 53,1 & 53,8 \\
$\mathbf{1 9}$ & 53,1 & 52,1 & 52,8 \\
$\mathbf{2 0}$ & 52,2 & 51,2 & 51,9 \\
$\mathbf{2 1}$ & 51,3 & 50,3 & 51,0 \\
$\mathbf{2 2}$ & 50,3 & 49,3 & 50,1 \\
$\mathbf{2 3}$ & 49,4 & 48,4 & 49,1 \\
$\mathbf{2 4}$ & 48,5 & 47,5 & 48,2 \\
$\mathbf{2 5}$ & 47,6 & 46,6 & 47,3 \\
$\mathbf{2 6}$ & 46,7 & 45,7 & 46,4 \\
$\mathbf{2 7}$ & 45,8 & 44,8 & 45,5 \\
$\mathbf{2 8}$ & 44,9 & 43,8 & 44,6 \\
$\mathbf{2 9}$ & 44,0 & 42,9 & 43,7 \\
$\mathbf{3 0}$ & 43,1 & 42,0 & 42,8 \\
$\mathbf{3 1}$ & 42,2 & 41,1 & 41,9 \\
$\mathbf{3 2}$ & 41,4 & 40,2 & 41,0 \\
$\mathbf{3 3}$ & 40,5 & 39,3 & 40,1 \\
$\mathbf{3 4}$ & 39,6 & 38,4 & 39,2 \\
$\mathbf{3 5}$ & 38,7 & 37,5 & 38,3 \\
$\mathbf{3 6}$ & 37,8 & 36,7 & 37,4 \\
$\mathbf{3 7}$ & 36,9 & 35,8 & 36,6 \\
$\mathbf{3 8}$ & 36,0 & 34,9 & 35,7 \\
$\mathbf{3 9}$ & 35,2 & 34,0 & 34,8 \\
$\mathbf{4 0}$ & 34,3 & 33,2 & 33,9 \\
$\mathbf{4 1}$ & 33,4 & 32,3 & 33,1 \\
$\mathbf{4 2}$ & 32,5 & 31,5 & 32,2 \\
$\mathbf{4 3}$ & 31,7 & 30,6 & 31,4 \\
$\mathbf{4 4}$ & 30,8 & 29,8 & 30,5 \\
$\mathbf{4 5}$ & 30,0 & 29,0 & 29,7 \\
$\mathbf{4 6}$ & 29,1 & 28,1 & 28,9 \\
$\mathbf{4 7}$ & 28,3 & 27,3 & 28,0 \\
$\mathbf{4 8}$ & 27,5 & 26,5 & 27,2 \\
$\mathbf{4 9}$ & 26,7 & 25,7 & 26,4 \\
$\mathbf{5 0}$ & 25,9 & 24,9 & 25,6 \\
\hline
\end{tabular}

\begin{tabular}{|c|c|c|c|}
\hline Edad & Mujeres & Hombres & Total \\
\hline 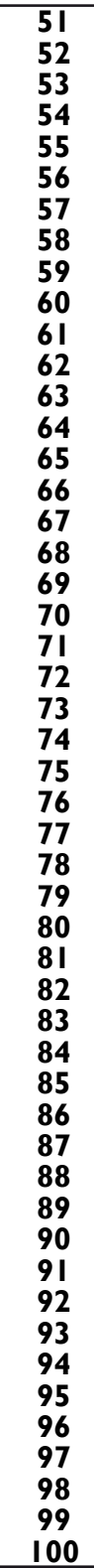 & $\begin{array}{l}25,1 \\
24,3 \\
23,5 \\
22,7 \\
21,9 \\
21,1 \\
20,4 \\
19,6 \\
18,9 \\
18,1 \\
17,4 \\
16,6 \\
15,9 \\
15,2 \\
14,5 \\
13,8 \\
13,1 \\
12,4 \\
11,8 \\
11,1 \\
10,5 \\
9,9 \\
9,3 \\
8,8 \\
8,2 \\
7,7 \\
7,2 \\
6,7 \\
6,3 \\
5,9 \\
5,5 \\
5,1 \\
4,7 \\
4,4 \\
4,1 \\
3,8 \\
3,5 \\
3,2 \\
3,0 \\
2,7 \\
2,5 \\
2,3 \\
2,2 \\
2,0 \\
1,9 \\
1,7 \\
1,6 \\
1,5 \\
1,4 \\
1,2\end{array}$ & 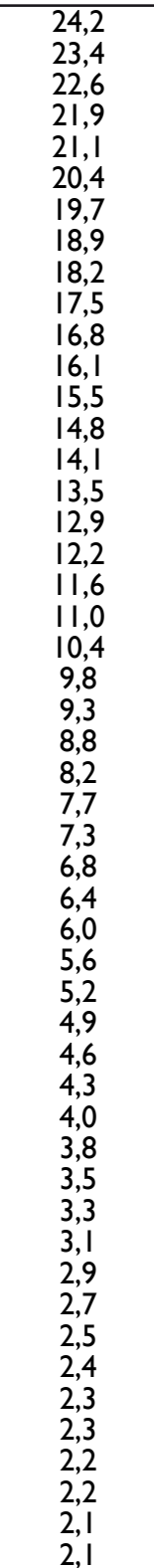 & $\begin{array}{l}24,8 \\
24,1 \\
23,3 \\
22,5 \\
21,8 \\
21,0 \\
20,2 \\
19,5 \\
18,8 \\
18,1 \\
17,3 \\
16,6 \\
15,9 \\
15,2 \\
14,5 \\
13,9 \\
13,2 \\
12,6 \\
11,9 \\
11,3 \\
10,7 \\
10,1 \\
9,5 \\
9,0 \\
8,5 \\
7,9 \\
7,5 \\
7,0 \\
6,5 \\
6,1 \\
5,7 \\
5,4 \\
5,0 \\
4,7 \\
4,3 \\
4,0 \\
3,7 \\
3,5 \\
3,2 \\
3,0 \\
2,8 \\
2,6 \\
2,4 \\
2,2 \\
2,1 \\
2,0 \\
1,8 \\
1,7 \\
1,6 \\
1,5\end{array}$ \\
\hline
\end{tabular}

Revista de Evaluación de Programas y Políticas Públicas | Núm. 6 (2016), pp. 28-48 


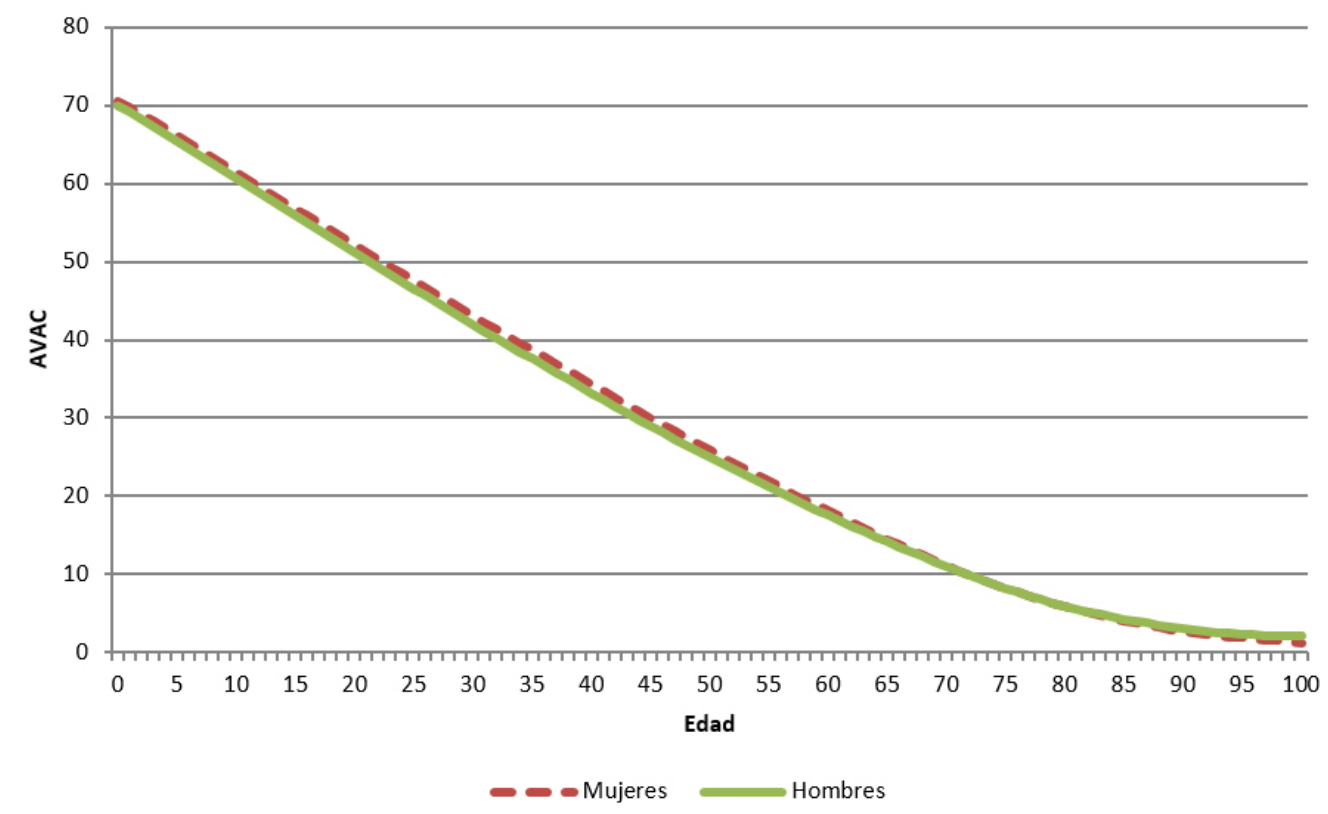

Gráfico 3. Esperanza de vida ajustada por la calidad por sexo y edad.

Las estimaciones mostradas hasta ahora no tienen en cuenta la existencia del fenómeno de preferencia temporal. Si asumimos que el valor que se otorga a los años de vida más lejanos en el tiempo es inferior al otorgado a los años más próximos, hemos de aplicar una tasa de descuento al cálculo de la EVAC. En el Gráfico 4 se presentan los resultados de aplicar diferentes tasas de descuento (1,5\%, $3 \%$ y $5 \%$ ), sin diferenciar, en este caso, por sexos.

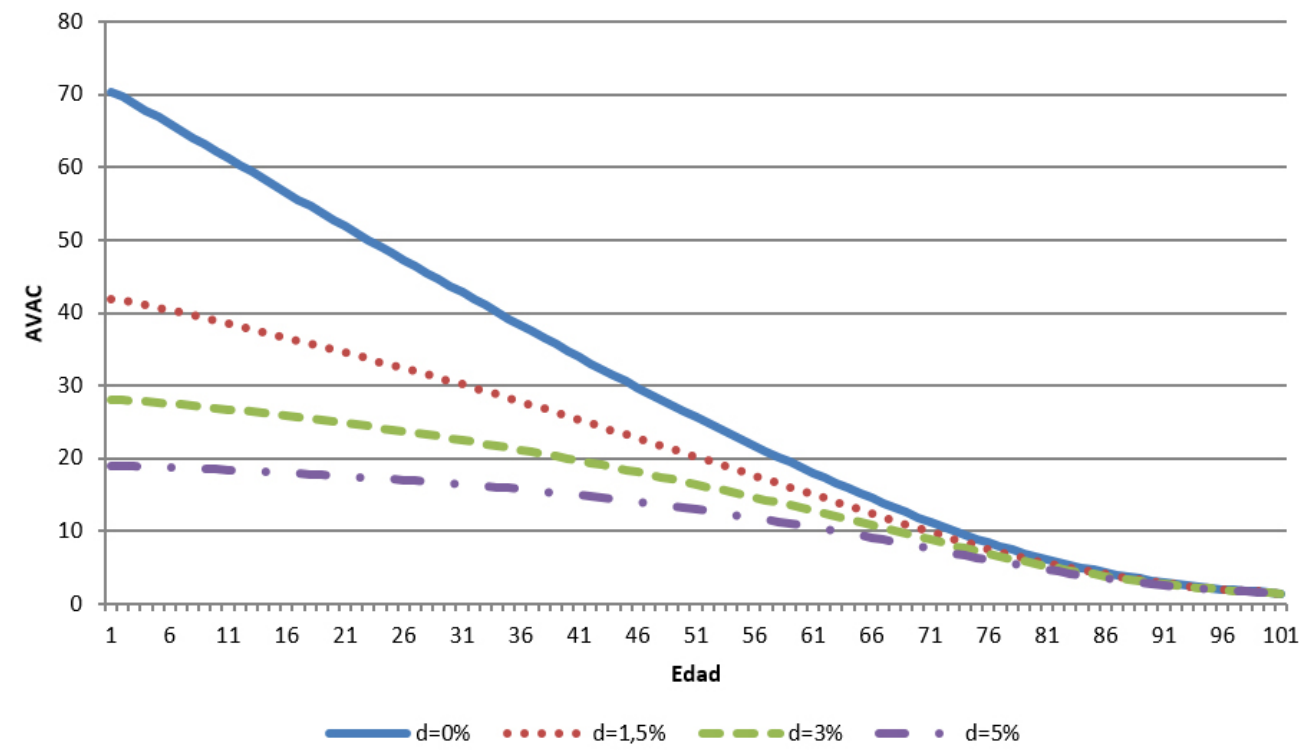

Gráfico 4. Valores de la Esperanza de vida ajustada por la calidad para distintas tasas de descuento (d) por edades; ambos sexos. 
Como es obvio, el valor descontado del flujo de años de vida será menor cuanto mayor sea la tasa de descuento. En consecuencia, las estimaciones de la EVAC al nacer, resultantes de aplicar cada una de las tasas de descuento ascienden a 41,9 AVAC, 28,1 AVAC y 18,9 AVAC, según se utilice una tasa del 1,5\%, del 3\% o del $5 \%$, respectivamente.

Como se señaló en la introducción, un segundo objetivo del estudio, junto al de obtener una primera estimación de la EVAC para España, era el de comparar nuestros resultados con las estimaciones disponibles a escala regional, con la intención de contrastar, de algún modo, la validez de éstas. Con el fin de que la comparación con algunas de estas estimaciones sea más homogénea, y habida cuenta de que los estudios que previamente estimaron la EVAC utilizando el mismo instrumento de medida de la CVRS que se ha empleado en este trabajo, esto es, el SF-6D, recurrieron al algoritmo de cálculo basado en preferencias de la población británica, hemos reestimado la EVAC con nuestros datos aplicando la tarifa del SF-6D para el Reino Unido. El Cuadro 5 muestra información de los distintos estudios que permite la comparación entre unos y otros resultados.

Cuadro 5. Comparación de las estimaciones de la EVAC para España con estudios previos realizados a escala autonómica.

\begin{tabular}{|c|c|c|c|c|c|c|c|c|}
\hline & \multirow{2}{*}{$\begin{array}{c}\text { Cataluña } \\
2002 \\
(a)\end{array}$} & \multirow{2}{*}{$\begin{array}{c}\text { Canarias } \\
2004 \\
\text { (b) }\end{array}$} & \multirow{2}{*}{$\begin{array}{l}\text { Cataluña } \\
2006 \\
\text { (c) }\end{array}$} & \multirow{2}{*}{$\begin{array}{c}\text { País Vasco } \\
2002 \\
\text { (d) }\end{array}$} & \multirow{2}{*}{$\begin{array}{c}\text { Andalucía } \\
2003 \\
\text { (e) }\end{array}$} & \multirow{2}{*}{$\begin{array}{c}\text { R. Murcia } \\
2009 \\
\text { (f) }\end{array}$} & \multicolumn{2}{|c|}{ ESPAÑA $201 \mathrm{I}$} \\
\hline & & & & & & & $\begin{array}{c}\text { Tarifa } \\
\text { SF-6D } \\
\text { española }\end{array}$ & $\begin{array}{c}\text { Tarifa } \\
\text { SF-6D } \\
\text { británica }\end{array}$ \\
\hline \multicolumn{9}{|l|}{15 años* } \\
\hline Hombres & 56,5 & $55, I$ & 57,3 & 51.6 & 55.1 & 52.1 & 55.9 & 57.2 \\
\hline Mujeres & 57,3 & 55,9 & 56,1 & 54.9 & 55.9 & 53.2 & 56.8 & 56.7 \\
\hline \multicolumn{9}{|l|}{25 años } \\
\hline Hombres & 48,0 & 45,8 & 47,9 & 43.2 & 45.8 & 43.7 & 46.6 & 47.6 \\
\hline Mujeres & 48,7 & 46,8 & 46,6 & 46.7 & 46.8 & 45.0 & 47.6 & 47.1 \\
\hline \multicolumn{9}{|l|}{35 años } \\
\hline Hombres & 38,9 & 36,7 & 38,5 & 34.9 & 36.7 & 35.5 & 37.5 & 38.1 \\
\hline Mujeres & 39,4 & 37,8 & 37,3 & 38.4 & 37.8 & 36.9 & 38.7 & 37.5 \\
\hline \multicolumn{9}{|l|}{45 años } \\
\hline Hombres & 30,2 & 27,9 & 29,6 & 26.9 & 27.9 & 27.5 & 29.0 & 29.1 \\
\hline Mujeres & 30,5 & 29,2 & 28,4 & 30.4 & 29.2 & 29.1 & 30.0 & 28.1 \\
\hline \multicolumn{9}{|l|}{55 años } \\
\hline Hombres & 22,1 & 20,3 & $2 I, 4$ & 19.6 & 20.3 & 20.1 & 21.1 & 21.0 \\
\hline Mujeres & 22,1 & 21,4 & 20,2 & 22.8 & 21.4 & 21.7 & 21.9 & 19.4 \\
\hline \multicolumn{9}{|l|}{65 años } \\
\hline Hombres & 14,7 & 13,7 & 14,2 & 13.1 & 13.7 & 13.3 & 14.1 & 13.5 \\
\hline Mujeres & 14,7 & 14,3 & 12,9 & 15.8 & 14.3 & 14.7 & 14.5 & 11.4 \\
\hline \multicolumn{9}{|l|}{75 años } \\
\hline Hombres & 8,8 & 7,9 & 8,0 & 7.6 & 7.9 & 7.8 & 8.2 & 7.2 \\
\hline Mujeres & 8,1 & 7,9 & 6,8 & 9.3 & 7.9 & 8.7 & 8.2 & 5.7 \\
\hline
\end{tabular}

* En la estimación de Cataluña (2002), los valores de la EVAC corresponden a los 16 años.

Nota: Las estimaciones de las tres primeras columnas utilizan el EQ-5D para los ajustes de calidad de vida; las restantes emplean el SF-6D, al igual que se hace en el presente trabajo.

Fuente: (a) Zozaya et al. (2005). (b) Zozaya y Oliva (2007). (c) Oliva (2008). (d) Esnaola et al (2006). (e) Martínez et al. (2008). (f) Abellán et al. (20I2b). 
En primer lugar, se ha de señalar que la comparación entre las diferentes estimaciones no resulta del todo homogénea. Para empezar, las tres primeras estimaciones que se muestran en el cuadro 5 utilizaron como instrumento de medida de la CVRS el EQ-5D y no el SF-6D. En segundo lugar, para el caso de la comunidad autónoma andaluza la caracterización SF-6D se realizó a partir del SF-12 y, por tanto, los valores obtenidos no son totalmente comparables. Además, se ha de tener presente que los años de las estimaciones son diferentes. Hechas estas salvedades, se observa en el cuadro 4 como los valores obtenidos para el conjunto nacional son superiores a los estimados a escala regional hasta los 35 años de edad, mientras que a partir de ese momento las conclusiones difieren según el sexo. Mientras que en el caso de los varones, los valores de la EVAC que hemos estimado para el conjunto de España siguen claramente por encima de los observados en las comunidades autónomas objeto de comparación, para las mujeres, sin embargo, afloran diferencias notables en sentido contrario, que se ven, además, incrementadas conforme se avanza en la estructura de edades. Este fenómeno, probablemente, tiene mucho que ver con la caracterización de estados de salud obtenida para la muestra de mujeres de mayor edad, ya que cuando se emplea la tarifa española en lugar de la británica, dichas diferencias se mitigan notablemente. La razón de ello es que en la tarifa española, las utilidades asociadas a los estados de salud menos graves son, en general, más altas que en la británica. En este mismo sentido, por tanto, se puede ver la relevancia de emplear una tarifa u otra en el SF-6D, sobre todo para los grupos de mayor edad, donde las personas que se alejan de salud plena representan una proporción cada vez mayor.

\section{Conclusiones}

La sociedad española ha experimentado a lo largo del último siglo un importante avance en términos de longevidad, como lo demuestra el incremento tan notable registrado en la Esperanza de Vida. En este escenario, donde el margen para obtener nuevas ganancias en términos de cantidad de vida parecen ser cada vez más estrecho, cobra especial sentido poner el foco de interés en la calidad de vida que acompaña a dicho horizonte vital. La Esperanza de Vida Ajustada por la Calidad (EVAC) es un indicador que combina cantidad y calidad de vida, y que sirve para cuantificar la salud de las poblaciones, constituyendo, además, un instrumento potencialmente valioso en el contexto de la evaluación de programas y políticas públicas de muy diversa naturaleza. En España, lamentablemente, no se cuenta con estimaciones de la EVAC, principalmente porque, hasta la fecha, no se ha dispuesto de medidas de la calidad de vida relacionada con la salud de la población susceptibles de ser utilizadas a tal fin. Sí existen, sin embargo, estimaciones de la EVAC para diversas comunidades autónomas españolas, gracias a que las encuestas regionales de salud de algunos de estos territorios proporcionan el input necesario para calcular los pesos de calidad de vida que se requieren para el cómputo de la EVAC. Este trabajo pretende contribuir a paliar la carencia de estimaciones de la EVAC a escala nacional.

Los valores que aquí se presentan resultan de combinar la información procedente de las Tablas de vida de la población española ofrecidos por el INE con datos sobre calidad de vida relacionada con la salud procedentes de una encuesta elaborada por la Dirección General de Tráfico. Los pesos de calidad de vida se han obtenido a partir de la conversión de las respuestas al cuestionario $\mathrm{SF}-36$, un cuestionario validado, al sistema descriptivo 
multi-atributo SF-6D, aplicando un algoritmo de cálculo de utilidades basado en preferencias de la población española. Adicionalmente, se ha llevado a cabo una segunda estimación con los pesos de calidad de vida resultantes de aplicar el algoritmo británico, por ser éste el empleado en estudios previos.

Los resultados obtenidos indican que la EVAC al nacer en España se sitúa en 70,5 y 69,9 años de vida ajustados por la calidad (AVAC), para mujeres y hombres, respectivamente. Esta pequeña diferencia entre sexos contrasta con la brecha mucho más amplia (6 años) que se encuentra en el valor bruto (sin ajustar por calidad) de la esperanza de vida al nacer; y pone de manifiesto que la mejor calidad de vida que experimentan los varones, particularmente en las edades más avanzadas, compensa su menor horizonte vital. La comparación realizada con los resultados de estudios previos para comunidades autónomas españolas, como el País Vasco, Andalucía o Murcia, en términos relativamente homogéneos, muestra que los valores no difieren significativamente, si bien son algo superiores los de nuestra estimación para el total nacional a los de las estimaciones regionales en los grupos de menor edad; y algo inferiores, especialmente en el caso de las mujeres, en las edades más avanzadas. Factores como el periodo de referencia, el ámbito geográfico o el diferente nivel de representatividad de las muestras pueden estar detrás de las diferencias observadas.

El trabajo presentado no está exento de limitaciones que resulta obligado reconocer. Una primera limitación se deriva del tamaño de la muestra, que apenas supera las cuatro mil observaciones, frente a las más de 20.000 observaciones que incluye, por ejemplo, la Encuesta Nacional de Salud. Esto compromete la robustez de la estimación, en particular cuando se desagrega por edad y por sexo. En segundo lugar, la utilización de datos de CVRS declarada por los propios individuos presenta los problemas propios del uso de valoraciones subjetivas, por otra parte común a la práctica totalidad de estudios previos de esta naturaleza. En tercer lugar, la muestra utilizada solo recoge información sobre calidad de vida de la población adulta, lo que obliga a realizar imputaciones para los grupos de menor edad; en nuestro caso hemos asumido una misma calidad de vida para todos los sujetos de 18 años o menos. En un sentido similar, se ha supuesto que más allá de los 80 años no se producía un deterioro relevante en el estado de salud. Por otro lado, la muestra no incluía hogares colectivos, lo que, junto al supuesto de calidad de vida constante a partir de 80 años, puede dar lugar a que se esté sobrestimando la calidad de vida en los grupos de mayor edad y, en consecuencia, también el valor de la EVAC para estas edades.

Las estimaciones que se presentan en este artículo deberían considerarse una primera aproximación al cómputo de la EVAC en España. Es éste el primer trabajo que lleva a cabo esta estimación para el conjunto del país, y uno de los pocos que lo ha hecho a escala internacional, tras las estimaciones para Estados Unidos (Cutler y Richardson, 1997; 1998; 1999) y Suecia (Burström et al. 2001; 2003). Futuros trabajos deberán orientarse a superar las limitaciones reseñadas. La disponibilidad de información sobre calidad de vida relacionada con la salud de la población española descrita con el instrumento EQ-5D-5L en la última Encuesta Nacional de Salud constituye una oportunidad para estimar la EVAC con información de una muestra de más amplio tamaño y con pesos de calidad de vida distintos de los utilizados en el presente estudio. Sería sin duda interesante comparar tales resultados con los que aquí se muestran. 


\section{Referencias Bibliográficas}

Abellán, J.M., Sánchez, F.I., Martínez, J.E. \& Méndez, I. (2012a). Lowering the floor of the SF-6D algorithm using a lottery equivalent method. Health Economics, 21, 1271-1285.

Abellán J.M., Martínez, J.E., Méndez, I., Sánchez, F.I. \& Garrido, S. (2012b). Financiación y eficiencia del sistema sanitario público de la Región de Murcia. Murcia: Consejo Económico y Social de la Región de Murcia.

Badía, X. \& Lizán,L. (2003). Estudios de calidad de vida. En A. Martín y J.F. Cano, Atención primaria: Conceptos, organización y práctica clínica. $5^{a}$ ed. (pp. 250261). Madrid: Elsevier.

Badía X., Fernández, E. \& Segura, A. (1995). Influence of sociodemographic and health status variables on valuation of health states in a Spanish population. European Journal of Public Health, 5, 87-93.

Badía, X., Roset, M., Herdman, M. \& Kind,P. (2001). A comparison of United Kingdom and Spanish general population time trade-off values for EQ-5D health states. Medical Decision Making, 21, 7-16.

Brazier, J., Usherwood, T., Harper, R. \& Thomas, K. (1998). Deriving a preference-based single index from the UK SF-36 health survey. Journal of Clinical Epidemiology, 51(11), 1115-1128.

Brazier, J., Roberts, J., \& Deverill,M. (2002). The estimation of a preference based measure of health from the SF-36. Journal of Health Economics, 21, 271-292.

Brazier, J.E. \& Roberts, J. (2004). The estimation of a preference-based measure of health from the SF-12. Medical Care, 42, 851-859.

Brazier J, Roberts J, Tsuchiya A, \& Busschbach J. (2004) A comparison of the EQ-5D and SF-6D across seven patient groups. Health Economics, 13, 873-884.

Cutler, D.M. \& Richardson, E. (1997).Measuring the health of the US population, Brooking papers on economic activity. Microeconomics, 21872, 217-271.

Cutler, D.M. \& Richardson, E. (1998). The value of health: 1979-1990. American Economic Review Papers Proceedings, 88, 97-100.

Cutler, D.M. \& Richardson, E. (1999). Your money and your life: the value of health and what affects it. NBER Working Paper Series, 6895, 1-75.

Dirección General de Tráfico (2010). El valor monetario de una vida estadística en España. Observatorio de Seguridad Vial. Madrid: DGT.

Dirección General de Tráfico (2011). El valor monetario de una víctima no mortal y del año de vida ajustado por la calidad en España. Observatorio de Seguridad Vial. Madrid: DGT. 
Esnaola, S., Unai S., Perez, Y., Ruiz, R, Alasoro, E. \& Calvo,M. (2006), Magnitud y desigualdades socioeconómicas de la esperanza de vida ajustada por calidad en la Vitoria: Departamento de Sanidad del Gobierno Vasco.

Fanshel, S. \& Bush, J.W. (1970). A health status index and its application to healthservices outcomes. Operational. Research, 18, 1021-1066.

García-Altés, A., Pinilla, J. \& Salvador, P. (2006). Aproximación a los pesos de calidad de vida de los «años de vida ajustados por la calidad» mediante el estado de salud autopercibido. Gaceta Sanitaria, 20, 457-464.

Gutiérrez, R., Linhart, J. \& Pitblado, J. (2003). From the help desk: local polynomial regression and Stata plugins. The Stata Journal, 3(4), 412-419.

Janssen, M.F., Pickard, A.S., Golicki, D., Gudex, C., Niewada, M., Scalone, L., ... Busschbach, J. (2013). Measurement properties of the EQ-5D-5L compared to the EQ-5D-3L across eight patient groups: a multi-country study. Quality of Life Research, 22, 1717-1727.

Martínez, J.E., Abellán, J.M. \& Pinto, J.L. (2008) El capital social de la salud en Andalucía. Sevilla: Junta de Andalucía.

Martínez, J.E., Sánchez, F.I., Abellán, J.M. \& Pinto, J.L. (2015). La valoración monetaria de los costes humanos de la siniestralidad vial en España. Gaceta Sanitaria, 29(S1), 76-78.

Ministerio de Sanidad y Política Social (2009). Indicadores de salud 2009. Evolución de los indicadores del estado de salud en España y su magnitud en el contexto de la Unión Europea. Madrid: MSPS.

Ministerio de Sanidad, Servicios Sociales e Igualdad (2013). Esperanzas de vida en salud en España 2006-2011. Años de vida saludable en España y sus comunidades autónomas. Madrid: MSSSI.

Murray, C., Salomon, J., Mathers, C. \& Lopez, A. (eds) (2002). Summary measures of population health: concepts, ethics, measurement and applications. Geneva: World Health Organization.

Nadaraya, E. A. (1964). On estimating regression. Theory of Probability and Its Application, 9, 141-142.

Oliva, J. \& Zozaya,N. (2007). Valoración y determinantes del stock de capital salud en la Comunidad Canaria y Cataluña. Documento de Trabajo de FEDEA 2007-29. http://documentos.fedea.net/pubs/dt/2007/dt-2007-29.pdf

Oliva, J. (2008). Valoración y determinantes del stock de capital salud en Cataluña: 19942006. Documento de trabajo de FEDEA. Serie Estudios de Economía Española o5-2008. http://www.fedea.net/documentos/pubs/ee/2008/05-2008.pdf

Oliva, J., Zozaya, N. \& González, B. (2010) Opposite poles: A comparison between two regions in health-related quality of life, with implications for health policy. BMC Public Health, 10, 576. 
Ramos-Goñi, J.M., Pinto-Prades, J.L., Oppe, M., Cabasés, J.M., Serrano-Aguilar, P. \& Rivero-Arias, O. Valuation and modeling of EQ-5D-5L health states using a hybrid approach. Medical Care. Postprint. DOI:10.1097/MLR.0000000000000283

Sanders, B.S. (1964) Measuring community health levels. American Economic Review, $54,1063-1070$.

Sullivan, D. F. (1971). A single index of mortality and morbidity. HSMHA Health Reports, $86,347-54$.

Ware, J.E. \& Sherbourne, C. (1992), The MOS 36-item short-form health survey (SF-36) (I). Conceptual framework and item selection, Medical Care, 30, 473-83.

Watson, G. S. (1964). Smooth regression analysis. Sankhya Series, A 26, 359-372.

Wilkins, R \& Adams, O.B. (1992). Quality-adjusted life expectancy: weighting of expected years in each state of health. En J.M. Robine, M. Blanchet \& J.E. Dowd (Eds.), Health expectancy, OPCS studies on medical and population subjects, 54 London: HMSO.

Zozaya, N., Oliva, J. \& Osuna, R. (2005). Measuring changes in health capital. Documentos de Trabajo de la Fundación de Estudios de Economía Aplicada, 2005-15. 